\title{
ANALYTIC TABLEAUX AND INTERPOLATION
}

\author{
Miodrag Kapetanović
}

\author{
Dedicated to Saša Kron, friend and techer.
}

\begin{abstract}
A tableau system for the predicate logic with countable conjunctions and disjunctions is presented and the completeness of the set of rules proved. These tableaux are used to prove a slightly more general form of the Malitz interpolation theorem.
\end{abstract}

\section{Introduction}

The logic $L_{\omega_{1} \omega}$, obtained from first order logic by allowing countable conjunctions and disjunctions, has been extensively studied and used in model theory ([3] is a standard reference). Although Gentzen systems for this logic also exist (see [4] but also [1] for a more recent treatment), the corresponding tableau system(s) do not seem to be used in practice. For that reason a tableau system for $L_{\omega_{1} \omega}$ is presented in some detail, followed by a completeness proof. Included are minor novelties in the inductive definition of tableau with possible technical advantages.

In order to simplify exposition all structures are assumed to have at least one constant. $L_{\omega_{1} \omega}$-formulas are defined as in the first order logic with the additional clause: if $\Phi=\left\{\phi_{i} \mid i<\omega\right\}$ is at most countable set of formulas, then both $\bigwedge \Phi$ and $\bigvee \Phi$ are formulas written also as $\bigwedge_{i<\omega} \phi_{i}, \bigvee_{i<\omega} \phi_{i}$ respectively. Hereafter a formula (sentence) will always mean an $L_{\omega_{1} \omega}$-formula (sentence). Writing $\varphi$ as $\varphi \mathbf{c y}$ we emphasize that all constants in $\varphi$ are among $\mathbf{c}=\left\{c_{0}, c_{1}, \ldots\right\}$ and that all free variables in $\varphi$ are among $\mathbf{y}=\left\{y_{0}, y_{1}, \ldots\right\}$ and similarly for sets of formulas.

\section{A tableau system for $L_{\omega_{1} \omega}$}

Since $L_{\omega_{1} \omega}$ is an extension of first order logic we could simply make use of the well known uniform notation of Smullyan [6], treating $\bigwedge \Phi$ as $\alpha, \bigvee \Phi$ as $\beta$, etc. Another approach is used instead, that seems simple and illustrative enough: only sentences in negation normal form, i.e., those which are built up from atomic formulas and their negations using $\wedge, \vee, \forall$ and $\exists$ are treated. There is no loss of generality since every formula is equivalent to a formula in such form, and $\varphi \wedge \psi$,

2000 Mathematics Subject Classification: 03C40, 03C75, 03 F99. 
$\varphi \vee \psi$ and $\varphi \rightarrow \psi$ are defined as $\bigwedge\{\varphi, \psi\}, \bigvee\{\varphi, \psi\}$ and $\bigvee\{\neg \varphi, \psi\}$ respectively. Hence the following rules are needed:

$$
(\wedge) \frac{\bigwedge \Phi}{\Phi} \quad(\vee) \frac{\bigvee_{i<\omega} \varphi_{i}}{\varphi_{0}\left|\varphi_{1}\right| \ldots\left|\varphi_{i}\right| \ldots} \quad(\forall) \frac{\forall x \varphi}{\varphi_{x} t} \quad(\exists) \frac{\exists x \varphi}{\varphi_{x} \mathrm{c}}
$$

Here $t$ is any ground term and $c$ is a new constant, which should be understood in the following sense. The set $L(\varphi)$ of all relational and functional symbols (including symbols of constants) occurring in $\varphi$ is the language of $\varphi$, and $L_{\mathrm{C}}(\varphi)$ is the expansion of $L(\varphi)$ by a countable set $\mathrm{C}$ of new constants, i.e., $L(\varphi)$ and $\mathrm{C}$ are disjoint, whereas by $\varphi$-term we mean any ground term generated by the constants from $L_{\mathrm{C}}(\varphi)$. More explicitly, if $f$ is an $n$-ary functional symbol from $L_{\mathrm{C}}(\varphi)$ and $t_{0}, \ldots, t_{n-1}$ a sequence of $\varphi$-terms, then $f t_{0} \ldots t_{n-1}$ is a $\varphi$-term.

Tableaux are defined as rooted trees whose nodes have at most countably many immediate successors and each node is labelled with a set of sentences. Nodes are denoted by $\sigma, \tau, \ldots$ and $\tau 0, \tau 1, \ldots$ are immediate successors of $\tau$. In the following definition $\mathbf{T}$ denotes a tableau for a given sentence $\varphi^{1}$ and $l_{\sigma}^{\mathbf{T}}$ is the label of the node $\sigma$ from the underlying tree of $\mathbf{T}$.

Definition 2.1. (1) A tree whose only node is labelled by $\{\varphi\}$ is a tableau for $\varphi$.

(2) If $\bigwedge \Phi \in l_{\sigma}^{\mathbf{T}}$, then $\mathbf{T}^{\prime}$ obtained from $\mathbf{T}$ by setting $l_{\sigma}^{\mathbf{T}^{\prime}}=l_{\sigma}^{\mathbf{T}} \cup \Phi$ is a tableau for $\varphi$.

(3) If $\forall x \psi \in l_{\sigma}^{\mathbf{T}}, t$ is a $\varphi$-term and if $\mathbf{T}^{\prime}$ is obtained from $\mathbf{T}$ by setting $l_{\sigma}^{\mathbf{T}^{\prime}}=$ $l_{\sigma}^{\mathbf{T}} \cup\left\{\psi_{x}(t)\right\}$, then $\mathbf{T}^{\prime}$ is a tableau for $\varphi$.

(4) If $\exists y \psi \in l_{\sigma}^{\mathbf{T}}$ and $\mathrm{c} \in \mathrm{C}$ is new for $\mathbf{T}$, then $\mathbf{T}^{\prime}$ is obtained from $\mathbf{T}$ by setting $l_{\sigma}^{\mathbf{T}^{\prime}}=l_{\sigma}^{\mathbf{T}} \cup\left\{\psi_{y} \mathrm{c}\right\}$ is a tableau for $\varphi$.

(5) Let $\tau$ be a leaf of a branch containing $\sigma$. If $\bigvee_{i<\omega} \varphi_{i} \in l_{\sigma}^{\mathbf{T}}$ and $\mathbf{T}^{\prime}$ stems from $\mathbf{T}$ by expanding the domain of $\mathbf{T}$ by $\{\tau i \mid i<\omega\}$ and by setting $l_{\tau i}^{\mathbf{T}^{\prime}}=\left\{\varphi_{i}\right\}$ for all $i<\omega$ then $\mathbf{T}^{\prime}$ (based on the new domain) is a tableau for $\varphi$.

For every leaf $\tau$ of the domain of $\mathbf{T}$ the set $\mathrm{B}=\bigcup\left\{l_{\sigma}^{\mathbf{T}} \mid \sigma\right.$ is a predecessor of $\left.\tau\right\}$ is a branch of $\mathbf{T}$. A branch is closed if it contains a pair of complementary closed literals and a tableau is closed if all its branches are closed. It is easily checked that the rules are correct: if a tableau for $\varphi$ closes, then $\varphi$ has no model.

Since Hintikka sets play the central role in completeness proofs we recall the definition and sketch the proof of the main lemma.

Definition 2.2. A set $\mathrm{H}$ of sentences is a Hintikka set if it satisfies the following conditions:

( $\mathrm{H} 0)$ for all atomic sentences $\phi$ from $L(\mathrm{H}),\{\phi, \neg \phi\} \nsubseteq \mathrm{H}$;

$(\mathrm{H} \wedge)$ if $\bigwedge \Phi \in \mathrm{H}$, then $\Phi \subseteq \mathrm{H}$;

$(\mathrm{H} \vee)$ if $\bigvee \Phi \in \mathrm{H}$, then $\Phi \cap \mathrm{H} \neq \emptyset$;

\footnotetext{
${ }^{1}$ There is no harm in speaking about tableaux for (at most) countable sets of sentences since any such set $\Phi$ can be replaced by $\Lambda \Phi$, or even about a tableau for a set of sets of sentences, where the intended meaning for $\left\{\Phi_{i} \mid i<\omega\right\}$ is $\bigvee\left\{\bigwedge \Phi_{i} \mid i<\omega\right\}$.
} 
$(\mathrm{H} \forall)$ if $\forall x \varphi \in \mathrm{H}$, then $\varphi_{x} t \in \mathrm{H}$ for all ground terms from $L(\mathrm{H})$;

$(\mathrm{H} \exists)$ if $\exists x \varphi \in \mathrm{H}$, then $\varphi_{x} t$ for some ground term $t$ from $L(\mathrm{H})$.

The connection is established by calling a closed non-literal used up on a branch if it satisfies the appropriate condition from the above definition. For instance $\bigwedge \Phi$ is used up on $\mathrm{B}$ if $\Phi \subseteq \mathrm{B}$ etc.

Lemma 2.1 (Hintikka's Lemma). Every Hintikka set possesses a term model.

Proof. Let $\mathcal{A}=\left(A, \ldots, f^{\mathcal{A}}, \ldots, \ldots, R^{\mathcal{A}}, \ldots\right)$, where $A=\{t \mid t$ is a ground term of $L(\mathrm{H})\}$ and $f^{\mathcal{A}}\left(t_{0}, \ldots, t_{n-1}\right) \stackrel{\text { def }}{=} f t_{0} \ldots t_{n-1}$ for all $n$-ary function symbols from $L(\mathrm{H}), n \in \omega$. We stipulate $\mathcal{A} \vDash \phi \Leftrightarrow \phi \in \mathrm{H}$ for all atomic sentences from $L(\mathrm{H})$ and the proof of $\mathcal{A} \vDash \mathrm{H}$ by induction on the complexity of sentences from $\mathrm{H}$ is straightforward. For instance if $\phi \in \mathrm{H}$ is atomic then $\mathcal{A} \vDash \phi$ by definition of $\mathcal{A}$ and if $\neg \phi \in \mathrm{H}$, then $\phi \notin \mathrm{H}$ by $(\mathrm{H} 0)$, so $\mathcal{A} \not \models \phi$ again by the definition of $\mathcal{A}$. Further if $\Lambda \Phi \in \mathrm{H}$, then $\Phi \subseteq \mathrm{H}$, so $\mathcal{A} \vDash \Phi$ by induction hypothesis, hence $\mathcal{A} \vDash \wedge \Phi$ and similarly for $\bigvee \Phi \in \mathrm{H}$, where $\mathcal{A} \vDash \Phi \cap \mathrm{H}$, again using induction hypothesis. If $\exists y \psi \in \mathrm{H}$, then $\psi_{y} t \in \mathrm{H}$ for some ground $t$. By induction hypothesis $\mathcal{A} \vDash \psi_{y} t$, so $\mathcal{A} \vDash \exists y \psi$. Finally if $\forall x \psi \in \mathrm{H}$, then $\psi_{x} t \in \mathrm{H}$ for all ground terms $t$, so $\mathcal{A}$ satisfies each of $\psi_{x} t$ by induction hypothesis and we may conclude $\mathcal{A} \vDash \forall x \psi$ since $\mathcal{A}$ is a term structure.

A set $\Gamma$ of sentences is consistent if there is no closed tableau for $\wedge \Gamma$.

Lemma 2.2. If $\{\varphi\}$ is consistent, then there is a Hintikka set containing $\varphi$.

Proof. We shall define a sequence $\mathbf{T}_{0}, \mathbf{T}_{1}, \ldots$ of tableaux for $\varphi$ together with the sequence $\mathrm{B}_{0} \subseteq \mathrm{B}_{1} \subseteq \ldots$ of respective consistent branches. Notice first that $L(\varphi)$ is countable for every sentence $\varphi$ and so is the set of all $\varphi$-terms as well as the set of all subsentences of $\varphi$ and only these occur in a tableau for $\varphi$. This altogether guarantees the countability of all sets of sentences appearing in the tableau construction. With this in mind we can easily devise a particular enumeration of all $L_{\mathrm{C}}(\varphi)$-sentences which ensures that the rule $(\forall)$ is applied to every (occurrence of a) sentence of the form $\forall x \psi$ unboundedly many times ${ }^{2}$. Starting with $\mathrm{B}_{0}=\{\varphi\}$ and using the fact that the rules are correct, we can show that, given consistent $\mathrm{B}_{i}$ from $\mathbf{T}_{i}$, any application of a rule must result in a $\mathbf{T}_{i+1}$ with at least one consistent branch $\mathrm{B}_{i+1} \supseteq \mathrm{B}_{i}$. As an illustration let $\exists y \psi \in \mathrm{B}_{i}$ and let $\mathrm{B}_{i+1}=\mathrm{B}_{i} \cup\left\{\psi_{y} \mathrm{c}\right\}$ (c new for $\mathbf{T}_{i}$ ). If $\mathrm{B}_{i+1}$ was inconsistent, then (by correctness) it would have no model, in other words $\mathrm{B}_{i} \vDash \neg \psi_{y} \mathrm{c}$ and, since $\mathrm{c}$ is new, $\mathrm{B}_{i} \vDash \forall y \neg \psi$, a contradiction since $\exists y \psi \in \mathrm{B}_{i}$. Other cases are similar so there are two possibilities: we either stop at some stage $i$ since all of $\mathrm{B}_{i}$ is used up or proceed generating a sequence $\left\{\mathrm{B}_{i} \mid i<\omega\right\}$. In the first case $B_{i}$ is obviously a Hintikka set,otherwise let $B=\bigcup_{i<\omega} B_{i}$. Notice first that $\{\phi, \neg \phi\} \nsubseteq \mathrm{B}$, since it would be included in some $\mathrm{B}_{i}$ which is impossible. Next, all sentences in B are obviously used up at some stage except universal ones. But given any such $\forall x \psi$ and any ground $t, \psi_{x} t$ must appear in some $\mathrm{B}_{i}$, since we kept visiting $\forall x \psi$ thus ensuring that all terms are attended to.

\footnotetext{
${ }^{2}$ It is understood that each time $\psi$ is instantiated by a term not used before for that occurrence of $\psi$.
} 
Reformulating the statement we get the main theorem.

Theorem 2.1 (Completeness Theorem). If $\vDash \varphi$, then there is a closed tableau for $\varphi$.

\section{Interpolation}

By $\mathcal{A}_{A}$ we mean the structure $\mathcal{A}$ enriched with all elements of $A$ as constants and its signature is denoted by $L_{\mathbf{a}}(\mathcal{A})$, where $\mathbf{a}$ is the list of names of all elements of $A$. Recall that $\operatorname{diag}(\mathcal{A})=\left\{\theta \mathbf{a} \mid \theta \mathbf{a}\right.$ is a closed literal and $\left.\mathcal{A}_{A} \vDash \theta \mathbf{a}\right\}$ is the diagram of $\mathcal{A}$.

THEOREM 3.1. Given a sentence $\sigma$ and a formula $\varphi \mathbf{z}$, suppose that for all $\mathcal{A}, \mathcal{B} \vDash \sigma$ and all $\mathbf{a} \subseteq A$, if $\mathcal{A} \subseteq \mathcal{B}$ and $\mathcal{B} \vDash \varphi \mathbf{a}$, then $\mathcal{A} \vDash \varphi \mathbf{a}$. Then there is a universal formula $\psi \mathbf{z}$ such that $\sigma \vDash \forall \mathbf{z}(\varphi \mathbf{z} \leftrightarrow \psi \mathbf{z})^{3}$.

Proof. Enrich $L$ with a set $\mathbf{c}=\left\{c_{0}, c_{1}, \ldots\right\}$ of new constants in 1-1 correspondence with $\mathbf{z}$. If $\psi_{\mathbf{z}} \mathbf{c} \equiv \bigwedge\left\{\chi_{\mathbf{z}} \mathbf{c} \mid \chi_{\mathbf{z}}\right.$ is an $L(\varphi)$-formula of the form $\forall \mathbf{x} \bigvee \Phi \mathbf{x z}$, where $\Phi$ is a set of literals and $\sigma, \varphi \mathbf{c} \vDash \chi \mathbf{c}\}$, then we shall prove that $\psi \mathbf{z}$ is the desired universal formula. Obviously $\sigma \vDash \varphi \mathbf{c} \rightarrow \psi \mathbf{c}$ and if the reverse is true we get $\sigma \vDash \varphi \mathbf{c} \leftrightarrow \psi \mathbf{c}$, hence $\sigma \vDash \forall \mathbf{z}(\varphi \mathbf{z} \leftrightarrow \psi \mathbf{z})$ with $\psi$ universal q.e.d.

Given $\mathcal{A} \vDash \sigma \wedge \psi \mathbf{c}$, it suffices to find $\mathcal{B} \supseteq \mathcal{A}$ such that $\mathcal{B} \vDash \sigma \wedge \varphi \mathbf{c}$, since by the theorem assumption $\mathcal{A} \vDash \varphi \mathbf{c}$. By the downward Löwenheim-Skolem theorem we may assume $\mathcal{A}$ countable, so enrich $L_{\mathbf{c}}(\varphi)$ with (the list of the names of) all elements of $A$ and let $\phi^{-}=\neg \phi$ and $(\neg \phi)^{-}=\phi$, for all atomic sentences $\phi$ of the expanded language. We claim that any tableau $\mathbf{T}$ for $\sigma \wedge \varphi \mathbf{c}$ (in this language) has a branch on which there is no literal $\phi \mathbf{a c}$ such that $\mathbf{a} \subset A$ and $\phi^{-} \mathbf{a c} \in \operatorname{diag}(\mathcal{A})$. Suppose not and let $\Phi \mathbf{a c}=\left\{\phi_{\mathrm{B}} \mathbf{a c} \mid \mathrm{B}\right.$ is a branch of $\mathbf{T}, \phi_{\mathrm{B}} \mathbf{a c} \in \mathrm{B}$ and $\phi_{\mathrm{B}}^{-} \mathbf{a c} \in$ $\operatorname{diag}(\mathcal{A})\}$. Then a tableau for $\sigma \wedge \varphi \mathbf{c} \wedge \wedge\left\{\phi^{-} \mathbf{a c} \mid \phi \mathbf{a c} \in \Phi \mathbf{a c}\right\}$ closes. This implies $\sigma \wedge \varphi \mathbf{c} \vDash \neg \wedge\left\{\phi^{-} \mathbf{a c} \mid \phi \in \Phi\right\}$, i.e., $\sigma \wedge \varphi \mathbf{c} \vDash \bigvee \Phi$ ac. Notice that a is finite and constants from a are new for $\sigma \wedge \varphi \mathbf{c}$, so $\sigma \wedge \varphi \mathbf{c} \vDash \forall \mathbf{x} \bigvee \Phi \mathbf{x c}$. Since $\mathcal{A} \vDash \sigma \wedge \psi \mathbf{c}$, it follows that $\mathcal{A} \vDash \forall \mathbf{x} \bigvee \Phi \mathbf{x c}$, hence $\mathcal{A} \vDash \bigvee \Phi \mathbf{a c}$, so $\mathcal{A} \vDash \phi$ ac for some $\phi \mathbf{a c} \in \Phi \mathbf{a c}$, contradiction since $\phi^{-}$ac $\in \operatorname{diag}(\mathcal{A})$. Together this implies that $\sigma \wedge \varphi \mathbf{c} \wedge \wedge \operatorname{diag}(\mathcal{A})$ is consistent, therefore has a model $\mathcal{B}$. As $\mathcal{B} \vDash \operatorname{diag}(\mathcal{A})$ we are done.

The above result is presented relative to a given sentence $\sigma$ in order to gain some generality. If we drop that condition we get the well known preservation theorem of Malitz [5], although he had it for universal sentences only. In fact somewhat more general result holds as well, one that resembles so called Malitz interpolation theorem, also proved in [5] (and again for sentences only).

THEOREM 3.2. Given a sentence $\sigma$ and formulas $\varphi \mathbf{z}, \theta \mathbf{z}$ suppose that for all $\mathcal{A}, \mathcal{B} \vDash \sigma$ and all $\mathbf{a} \subseteq A$, if $\mathcal{A} \subseteq \mathcal{B}$ and $\mathcal{B} \vDash \varphi \mathbf{a}$, then $\mathcal{A} \vDash \theta \mathbf{a}$. Then there is a universal formula $\psi \mathbf{z}$ such that $\sigma \vDash \forall \mathbf{z}(\varphi \mathbf{z} \rightarrow \psi \mathbf{z}) \wedge \forall \mathbf{z}(\psi \mathbf{z} \rightarrow \theta \mathbf{z})$.

Proof. Define $\psi$ as above and repeat the whole argument, only that this time we conclude $\mathcal{A} \vDash \theta \mathbf{c}$, so that $\sigma \vDash \psi \mathbf{c} \rightarrow \theta \mathbf{c}$ and we are done.

\footnotetext{
${ }^{3}$ It is fairly obvious that the condition is necessary as well.
} 


\section{Conclusion and future prospects}

We have presented a tableau system for the predicate logic $L_{\omega_{1} \omega}$ resembling Gentzen systems and applicable in model theory. Beside completeness proof a kind of interpolation theorem is proved that generalizes a result of Malitz using only a simple notion of diagram. It seems that a refined version of the infinitary case of the omitting types theorem can be obtained in the same manner. On the effective side, we can go back to the first order case and look for more effective constructions, leading possibly to automated treatment, since the form of the main results is retained.

\section{References}

[1] H.-D. Ebbinghaus, J. Flum, W. Thomas, Mathematical Logic, Springer-Verlag, New York, 1984

[2] W. Hodges, Model Theory, Cambridge University Press, 1993

[3] H. J. Keisler, Model Theory for Infinitary Logic, North-Holland, Amsterdam, 1971

[4] E. G. K. Lopez-Escobar, An interpolation theorem for denumerably long formulas, Fund. Math. 57 (1965), 254-272

[5] J. Malitz, Universal classes in infinitary languages, Duke Math. J. 36 (1969), 621-630

[6] R. Smullyan, First-Order Logic, Dover, New York, 1995

Matematički institut

Kneza Mihaila 36

11000 Beograd, p.p. 367

Serbia

kapi@mi.sanu . ac . yu 